\title{
Pleasurable music selects for enhanced music memory, hence music emotions: The evolutionary forces laid bare
}

\author{
Mark S. Riggle \\ Causal Aspects LLC, Charlottesville, VA, USA \\ markriggle@alumni.rice.edu \\ Published 16 December 2021; https://doi.org/10.18061/FDMC.2021.0022 \\ Author video presentation and/or other conference material: https://doi.org/10.17605/OSF.IO/RYP7N
}

\begin{abstract}
We have a phenomenal memory for music and seem highly motivated to remember pleasurable music. Since emotions greatly enhance memory, perhaps music evoked emotions are responsible for our substantial music memory. This makes music an evolutionary difficulty because of that consumed memory plus music's strong pleasures drive resource consuming behaviors that appear not useful to survival. Why should music evoked emotions, which enhance memory, exist and be so pleasurable? We introduce a new evolutionary framework for music where an important mechanism has been overlooked: Trait elaboration for sensory exploitation of a sensory preference. The theory shows that music selection developed cognitive neural capacities that are directly reusable for providing language. Music is not a fitness indicator, but it is attractive which makes evolution run.
\end{abstract}

KEYWORDS: evolution, emotions, memory, dance, language

\section{Introduction}

When looking at theories for the evolutionary origin of music, sexual selection for music has been taken to mean that music evolved as a fitness indicator of male mate quality. This has been a huge error and missed an important mechanism for the evolution of music; that is, sexual selection by female choice could have occurred for music (and likely did) without it having anything to do with being a fitness indicator. Calling it sensory exploitation of a sensory preference would be clearer (Ryan, 1998). How can that work and why the confusion?

\section{Trait Elaboration for Sensory Exploitation}

First, by sexual selection on female choice, no trait can really start off selected as a fitness indicator because there are several steps that must occur before that trait can become an indicator. While those steps are usually (and reasonably) ignored by evolutionary biologists (because they want to explain an existing sexually dimorphic trait - such as the peacock tail), the steps are critical in the analysis for music selection because music is not sexually dimorphic. The steps for sexual selection start with two initial parts: one, an initial pre-existing sensory preference (based on food, colors, warmth, or whatever environmental factors attract and reward individuals); and two, sensory exploitation where males evolve traits (by genetic mutations) to exploit that preference. So far, no sexual dimorphism, no indicator, sexes are equal. The details of the mechanism are important to see how music could evolve.

A sensory preference will attract all individuals, both males and females, to a particular environment. Now, males, who by new genetic mutations express a trait which stimulates that preference, will have greater reproductive success - this is sensory exploitation - and they will have more offspring carrying those genes. However, the genes responsible for that trait are not sexlinked and will express the phenotype in both the male and female offspring -- the genes do not 'know' they were selected by providing an advantage to a male. This selection will continue creating a stronger expressed (or more elaborated) trait(s) (and expressed in both males and females) until the expressed trait causes a reduced fitness to females. That is, while the males gain a reproductive advantage from the trait, females do not and will have a different cost/benefit tradeoff.

The critical point occurs when, for females, the trait's fitness costs exceed its benefits; at that point, natural selection will sex-link the trait to reduce its phenotypic expression in females (for example, a mutation occurs where testosterone presence regulates the trait's phenotypic expression - it turns the gene on, perhaps at puberty); So reducing the trait's expression in females will now increase the female's fitness. This sex-linking is when that trait becomes sexually dimorphic and sexual selection (sensory exploitation) can now continue to develop the trait at an exponential rate; this is because both sexes of offspring, now dimorphic, have increased fitness while carrying the same genotype.

For the evolutionary biologists, the next step of interest is why females maintain the strong preference for the displayed sexually dimorphic trait. We are finally at that last step to the trait being a fitness indicator: The 
female preference for the trait (the pleasure from) is maintained by natural selection only if the female gains in fitness (by more fit offspring) by keeping that preference. That is, the now sexually dimorphic male trait now indicates 'good-genes' - a fitness indicator.

The critical point is, if a trait provides fitness benefits to the female that exceeds the trait's costs, the trait will not become sex linked. That is, until the genes are sexlinked for expression, the trait, even though providing a reproductive advantage to males, will not be sexually dimorphic; thus, the trait cannot be a fitness indicator, and it cannot be even a sexual display.

\section{Applying This Principle to Music}

We can apply this principle to music, which is many traits and genes, and clearly creates strong sensory preferences (pleasures), and incurs high costs. Music capabilities can be developed by sensory exploitation by males, but still not be a fitness indicator, nor be sexually dimorphic, nor be a sexual display (thus even children will make and enjoy music, social groups will make music, and mothers will sing to infants because infants find it pleasing); these musical trait developments remain non-sex-linked and sexually monomorphic if and only if the music traits provide counterbalancing benefits to females.

\section{Counterbalancing Benefits from the Music Traits}

Those counterbalancing benefits fall into two categories: 1) the music traits develop the cognitive apparatus that can be reused for language (both males and females benefit greatly); 2) they provide infant protection from male conspecifics by female bonding [1].

For music, we have to show various elements for this framework:

1. the nature of the preexisting sensory preference that starts the selection process and what musiclike traits can exploit that initial preference

2. the additional cycles of new preferences and new exploitation traits that will occur for music

3. how those traits produce the cognitive apparatus usable for language which is the huge benefit offsetting the costs of the music traits - this narrows the concepts of what is language

4. why the various traits and pleasures of music are selected and developed including emotions induced by music (emotions were selected to enhance memory for music - and why that enhanced fitness)
Before going into these framework details, we need to discuss some of aspects of language and why the evolved music traits may provide the foundation for language (this is not by a music protolanguage).

\section{Language - What Is It and Why So Useful?}

The major issue for the origin of language (with a major simplification of the first extreme) is between the biolinguistic approach and the usage-based approach.

1. Biolinguistic approach - Is language an innate, syntax-specific, genetically endowed, 'language instinct', produced by evolution for communication (or even just some lesser combination of those elements)? Or the:

2. Usage-based approach -- Is language an emergent phenomenon based on latent cognitive capabilities where language is a learned skill in which linguistic structure is a product of culture (Christiansen \& Chater, 2016)?

The first approach has been described (perhaps a little unfairly) as "biologically implausible, unresponsive to cross-linguistic facts, theoretically inelegant, and unnecessary from the perspective of language acquisition". Of course, it is the latter view of language that fits with the music evolution framework here: where language is based on latent cognitive capabilities, a learned skill from the surrounding culture, and language acquisition occurs by building up a set of mental procedures for understanding and producing language (Christiansen \& Chater, 2016). An important question is why do those required cognitive capabilities seem to exist only in humans? We will show that the music framework may have created those cognitive capabilities that are needed for language. In this view, language is much more than just grammar and speech (there are more than a million published articles on aspects of language), rather, language is a powerful cognitive tool for problem solving, for reasoning about the world - and we know knowledge is power. Communication is not the driving force for language; however, the surrounding culture holds the knowledge to perform and use language to great effect. Therefore, communication is needed to learn language: to learn vocabulary, categories, and category features necessary for reasoning. This powerful problem-solving tool of language is the huge benefit that counterbalances the huge costs of music (and hence music stays monomorphic between the sexes). 


\section{Going from Music to Language and Cognition}

Music and language - a truly vast literature exists on how they overlap but not for the context of sensory exploitation with music selecting cognitive traits and where language is purely reusing those cognitive capabilities. Music in this framework could not be one genetic change but was many steps where each music capacity could grow in capability by additional genetic change. Similarly, because language is riding on those capacities, language would also grow in capability and grow in its useful power of reasoning and problem solving. Neither music nor language could be binary.

In sensory exploitation by males, any music trait that allows creating more a pleasurable music experience will attract females, which gives those males better reproductive success (making those genes spread in the population). Importantly, as long as that trait also supports language or other benefits (cognitive or infant protection), the trait will not become sex-linked and will express in all individuals: male, female, young, old, and will not become a sexual display. Furthermore, because that music trait is also expressed in females, it will change the sensory preference (or pleasures) from music; this feedback loop will strongly drive a growing complexity of many music traits (a classic runaway that maximizes the traits' development). The most influencing music traits would be the pleasure producing ones since they create that positive feedback loop for further sensory exploitation.

Of course, a large difference between language and music is the varied and intense pleasures of music, which seem absent or mostly absent from language. Music pleasure is dopamine related (Cardona et al., 2020) for example), and the more dopamine, the greater the reward. However, there is not just one monolithic music pleasure, there are multiple music pleasures that have originated over time. Some of the pleasures are: dancing to music or to rhythmic sounds; listening to music for the anticipation pleasure of music; learning new music; creating music; the emotions evoked by music; and music chills.

Each of these pleasures allow sensory exploitation and require cognitive developments to produce both the pleasure and the exploitation skill. Furthermore, those particular cognitive developments must then support a non-music benefit (cognitive, language related or infant protection) or else become sex-linked and sexually dimorphic. A brief and incomplete analysis of these pleasures follows.

\section{Dancing Pleasure}

Dancing is very pleasurable (Bernardi et al., 2017) and even for children. The pleasure of dancing to rhythmic sounds is very likely the first step in this music evolution, a pleasure that began independent from music (see its own section). Thus, before any music-like trait existed, because of sensory exploitation, dancing pleasure will give males who can create interesting rhythmic sounds a reproductive advantage. Creating those sounds requires some new cognitive abilities - a pattern generating ability and a memory for the created patterns and a memory for remembering the patterns produced by other individuals -- one can make more pleasing patterns by learning them from others. Additionally, a motivation to create rhythmic sounds is required, thus another trait under selection is a pleasure from making those sounds. [One benefit to females for creating those sounds is social bonding with other females to protect infant offspring.]

\section{Anticipation Pleasure of Music -- Needing Memory}

The pleasure of listening to music given by the music anticipation pleasure would have built on the prior pattern generation and the remembering of the patterns. This would become the 'rules' system for music along with the statistical analyses of auditory input and sensitivity to abstract patterns. This is the expectation system (Huron, 2006) which requires remembering much music, including the rules used during listening. The rules are acquired through mere listening to music, and those acquired rules are also used in creating music. Since not all music is pleasurable to listeners (culturally based), and because of the sensory exploitation principle, the learned rule system would be structured to remember pleasurable music. It learns what are pleasing music rules and uses them; as predicted, the greater the pleasure from music (more dopamine), the better its 'musical-memory' after a memory consolidation period (Cardona et al., 2020). This system will have been under selection through many generations producing greater capability in pattern generation, memory, and pleasure selectivity for creating more pleasurable music. This increase in pattern generation requires greater demands on the memory system for remembering music and its many (abstract) rules. For a review, see Ettlinger et al. (2011).

The benefit of the music rules system to language reuse is direct. Language learning is the building up of a set of procedures (rules) for understanding and producing language (Christiansen \& Chater, 2016); this includes memory intensive acquiring of vocabulary, 
categories, features and their relationships. As with the music elements, these language elements are also acquired from the surrounding culture of people. This language use is exactly like the learning of music rules and how memory for rules and pattern extraction is structured. Language development and use follows from the processing rules and regularities for the mapping between meanings and signals in the cognitive system provided by the music capabilities.

\section{Pleasure from Emotions Evoked by Music}

The pleasure from emotions evoked by music and from music chills become understandable in this framework; the memory requirements for learning the rules and patterns of music are huge, and emotions greatly enhance memory. Have a better and faster memory for music and the music rules system will then allow acquiring more rules (from listening to others); thus creating more pleasurable music. Hence, sensory exploitation will select for any traits that improve learning new music rules from other people; one of those traits is a better memory for music. Since emotions enhance memory greatly [happy and sad emotions use different memory systems (Kensinger \& Kark, 2018)], all emotional memory systems will eventually get selected for enhancing music memory -- memory for both what was heard veridically and the rules used in the anticipation. This means music is not for communicating emotions, but rather emotions are felt as a side effect of using them for memory enhancement (happy and sad music to the two memory systems). Furthermore, those music emotional responses will be selected to be pleasurable in order to motivate their important memory enhancing use.

Music chills are an extension of that memory enhancement by adding additional dopamine, epinephrine and cortisol, which strongly enhance memory (Kensinger \& Kark, 2018). Dopamine is critical in memory formation and is strongly present in the music evoked emotions (Cardona et al., 2020), as predicted here.

A problem is that the music evoked emotions appear specific to music and do not seem to occur with language in general. Hence, what is the benefit to language that is needed to prevent sex-linking of the traits for music emotion? The answer seems to be the exercising of the rule acquisition system with memory for what is heard along with the particular anticipation rules used. That is, this complex music rules system needs to acquire music rules developmentally in order to process language well; the pleasurable music emotions motivate practice on complex auditory music to hone the necessary learning skills; skills needed for language acquisition and use. This is supported by studies showing that music exposure may be needed to learn language well (Brandt et al., 2012). For example, informal music exposure at home impacts the development of complex language skills in 3 and 4year-old children (Politimou et al., 2019).

Interestingly then, young children must easily experience music evoked emotions and chills.

\section{Why should dancing be pleasurable?}

If the pleasure from dance is the initial sensory preference that can start the evolutionary cycle for music, where and why did the pleasure arise?

For humans, is moving in time to a beat an innate capability (perhaps a side-effect of vocal learning [2]) or is it a learned skill because some sensory sensation of dance is internally rewarded? We claim the latter. Dance is movement where the foot hits the ground or the head nods in time to music beats. Both of those movements will produce a vestibular jolt in time with an audible, rhythmic, musical beat. If some neural mutation caused that sensation to be internally rewarded - a Rhythmic Audible Vestibular Jolts (RAVJ) reward - dance would be rewarded and learned. Importantly, that mutation would have also produced human bipedalism; an advantage which would forever maintain that mutation (Riggle, 2018).

There is data supporting a RAVJ reward for motivating learning to dance (or operant conditioning). Infants (9-13 months old) will move to strongly rhythmic music (Zentner \& Eerola, 2010); however, their movements are jerky as they fling their limbs outwards and have a rapid stop at full extension (see the authors' video segments). That fling and stopping motion will produce a vestibular jolt. Importantly, and why this study is so critical, their movements were rarely in time to the music (period and phase), but when they were in time (on the beat), they smiled more $(\rho<0.01)$. Because smiling in infants mean they like what is occurring, the sensation must be pleasurable for them. This is consistent with a strong RAVJ reward motivating beginning to learn to move (to produce a vestibular jolt) in time to music.

Additional support comes from dancing children: dancing two-year-olds go in and out of phase; four-yearolds are much better while six-year-olds are near perfect. This is consistent with a rewarded learned skill; years of practice may be needed, but they really like to 
dance. Hence, the RAVJ reward is consistent as the initial reward for dancing pleasure which started the sensory exploitation cycles for creating the human music abilities and the side-effect of language.

\section{Conclusion}

We have shown that sensory exploitation of a sensory preference will drive evolution for rapid elaboration of traits that exploit the preference. This process is the first half of sexual selection, but the second half where sexlinking the trait occurs can be held off (and thus not be a fitness indicator trait). We have shown music can be developed under that evolutionary process as long as its developing cognitive machinery is also usable for language or other benefit to females.

Because pleasurable music is a sensory preference, the many developed cognitive abilities and pleasures for music are expected by a feedback loop of sensory exploitation. This theory explains music pleasures - why they exist and why there are so many. Additionally, this is the only theory that predicts music evoked emotions that enhance memory (and explains why happy and sad music emotions exist) and explains why music emotions are so prevalent and pleasurable (by dopamine). Furthermore, this theory explains why music, with emotions, interacts with language development, and why music exposure can improve language use in children.

We have shown music was not selected for communicating emotions (hard to show why that would be a benefit); in fact, music is not a communication system at all. However, because the emotional responses are selected to be so strong (for memory enhancement), human culture has figured out how to exploit that pleasurable emotional memory response (such as in film music). We also showed that language is consistent as an emergent capability based on the cognitive assets developed for music processing. This implies that language is also not for communication but supplies a benefit of a reasoning tool. Just as with music, language development needs to acquire the knowledge from the surrounding culture, hence the communication aspects of language.

Finally, we have shown that a simple neural reward mechanism for RAVJ may have started the dance pleasure which triggers the music evolution cycles.

\section{Endnotes}

1 Infanticide by males is common in chimpanzees but rare in bonobos. Bonobo females socially bond using sexual activity; hence, social bonding from dancing may have also prevented infanticide.

${ }^{2}$ Some issues on the vocal learning hypothesis: it does not explain the smiling babies when jolts are in time to the music -- the Zentner phenomena; why dance produces vestibular jolts; entrainment is a behavior and thus needs a reward motivation, left unexplained; why years are needed to become proficient; and the actual required relationship to language vocal use is unclear.

\section{References}

Bernardi, N. F., Bellemare-Pepin, A., \& Peretz, I. (2017). Enhancement of Pleasure during Spontaneous Dance. Frontiers in Human Neuroscience, 11. https://doi.org/10.3389/fnhum.2017.00572

Brandt, A. K., Slevc, R., \& Gebrian, M. (2012). Music and Early Language Acquisition. Frontiers in Psychology, 3. https://doi.org/10.3389/fpsyg.2012.00327

Cardona, G., Rodriguez-Fornells, A., Nye, H., Rifà-Ros, X., \& Ferreri, L. (2020). The impact of musical pleasure and musical hedonia on verbal episodic memory. Scientific Reports, 10(1), 16113. https://doi.org/10.1038/s41598$\underline{020-72772-3}$

Christiansen, M. H., \& Chater, N. (2016). Creating Language: Integrating Evolution, Acquisition, and Processing. MIT Press. https://doi.org/10.7551/mitpress/10406.001.0001

Ettlinger, M., Margulis, E. H., \& Wong, P. C. M. (2011). Implicit Memory in Music and Language. Frontiers in Psychology, 2. https://doi.org/10.3389/fpsyg.2011.00211

Huron, D. (2006). Sweet anticipation: Music and the psychology of expectation (pp. xii, 462). The MIT Press. https://doi.org/10.7551/mitpress/6575.001.0001

Kensinger, E. A., \& Kark, S. M. (2018). Emotion and Memory. In Stevens' Handbook of Experimental Psychology and Cognitive Neuroscience (pp. 1-26). American Cancer Society. https://doi.org/10.1002/9781119170174.epcn101

Politimou, N., Dalla Bella, S., Farrugia, N., \& Franco, F. (2019). Born to Speak and Sing: Musical Predictors of Language Development in Pre-schoolers. Frontiers in Psychology, 10. https://doi.org/10.3389/fpsyg.2019.00948

Riggle, M. (2018). A simple neural mutation that generates reward from rhythmic audible vestibular jolts fully explains all aspects of human bipedal gait development, including cerebral palsy gaits. Program No.495.12. 2018 Neuroscience Meeting Planner. San Diego, CA: Society for Neuroscience, 2018. Online., 898. 
Ryan, M. J. (1998). Sexual Selection, Receiver Biases, and the Evolution of Sex Differences. Science, 281(5385), 1999-2003. https://doi.org/10.1126/science.281.5385.1999

Zentner, M., \& Eerola, T. (2010). Rhythmic engagement with music in infancy. Proceedings of the National Academy of Sciences, 107(13), 5768-5773. https://doi.org/10.1073/pnas.1000121107 